\title{
DORSAL DISLOCATION OF THE FIRST METATARSO-PHALANGEAL JOINT
}

\author{
REPORT OF FOUR CASES
}

\author{
A. C. Giannikas, G. Papachristou, N. Papavasiliou, P. Nikiforidis \\ and G. Hartofilakidis-Garofalidis, Athens, Greece
}

From the University Department of Orthopaedics, K.A.T. Accident Hospital, Kifissia, Athens

\begin{abstract}
The anatomy of the first metatarso-phalangeal joint and of dorsal dislocation of the phalan $x$ are described. As with similar lesions in the hand, closed reduction is impossible because of interposition of the volar plate. Open reduction is essential and should be performed as soon as possible after the injury.
\end{abstract}

Dislocation of the first metatarso-phalangeal joint is rare. Apart from a brief mention in several textbooks on traumatology we have been unable to find a full description of this disorder in the literature, and there has been no reference to it in either the American or the British volume of this Journal.

Over 10,000 patients were admitted to the Orthopaedic Clinic of Athens University during the period 1963 to 1973 , and four were found to have dorsal dislocation of the first metatarso-phalangeal joint. The injury is similar to dorsal dislocation of the metacarpo-phalangeal joint of the index and little fingers and presents similar problems. The toe is deformed and shortened, with the proximal phalanx extended and with its base displaced over the head of the first metatarsal; the distal phalanx is flexed. Closed reduction is unsuccessful, even if attempted immediately after the accident, and as with the index and little fingers, open reduction may be difficult unless the anatomy of the deformity is well understood.

\section{THE NORMAL ANATOMY OF THE FIRST METATARSO-PHALANGEAL JOINT}

This joint is formed by the rounded head of the first metatarsal bone and the cup-like depression of the base of the proximal phalanx (Gray 1967). The plantar portion of the capsule is reinforced by a fibrocartilaginous plate (plantar accessory ligament), similar to that in the thumb, in which are two sesamoid bones facing the head of the metatarsal. Distally the fibrous plate is firmly attached to the volar aspect of the base of the phalanx, while proximally it is loosely attached to the subcapital area of the metatarsal. The upper surface of the plate glides on the plantar surface of the head, while the inferior part, being grooved, contributes to the formation of the tendon sheath of flexor hallucis longus. The lateral margin fuses with the transverse metatarsal ligament. The joint is reinforced on both sides by the collateral ligaments, which are identical in their arrangement with their counterparts in the hand. The synovial membrane is quite lax.

\section{ANATOMY OF DORSAL DISLOCATION}

The dislocation is caused by severe hyperextension of the hallux when the plantar surface of the outstretched toe strikes a resistant object. The toe assumes a characteristic position. The proximal phalanx rests on the dorsum of the metatarsal head, in extension with some outward deviation. The distal phalanx is flexed and the extensor tendons are relaxed. There is a rounded prominence on the plantar surface of the joint formed by the head of the metatarsal under the stretched plantar skin.

Lateral radiographs show the head of the first metatarsal depressed into the sole of the foot and the proximal phalanx displaced on to the dorsum of the head of the metatarsal. The two sesamoids are found occupying the lateral aspect of the metatarsal-phalangeal joint and are usually not separated (Figs. 1 and 3). However, in one of our cases the sesamoids were dorsal to the neck of the metatarsal and widely separated (Fig. 2).

Because of acute hyperextension of the toe at the moment of injury, the plantar part of the capsule is avulsed from the junction of head and neck of the metatarsal; the base of the first phalanx slides over the head of the metatarsal followed by the fibrocartilaginous plate and becomes locked in this position. The collateral ligaments remain intact and maintain the locked fibrocartilaginous plate over the dorsum of the head of the metatarsal, while the flexor tendons assume a position lateral to the head of the metatarsal.

Closed reduction is clearly impossible, unless of course, the lateral ligaments have been torn.

\section{CASE REPORTS}

Case 1-A man aged fifty-eight sustained a typical dislocation from an injury to his left foot in a traffic accident in June 1967 (Fig. 1). On the same day an attempt at closed reduction failed.

Dr A. Giannikas, Associate Professor of Orthopaedics of University of Athens, 5 Heraklitou Street, Athens-136, Greece. Dr G. Papachristou, Registrar, Orthopaedic Clinic, Athens University Accident Hospital, Kifissia, Athens, Greece. Dr N. Papavasiliou, Registrar, Orthopaedic Clinic, Athens University Accident Hospital, Kifissia, Athens, Greece. Dr P. Nikiforidis, Athens University Accident Hospital, Kifissia, Athens, Greece.

Professor G. Hartofilakidis-Garofalidis, Athens University Accident Hospital, Kifissia, Athens, Greece. 

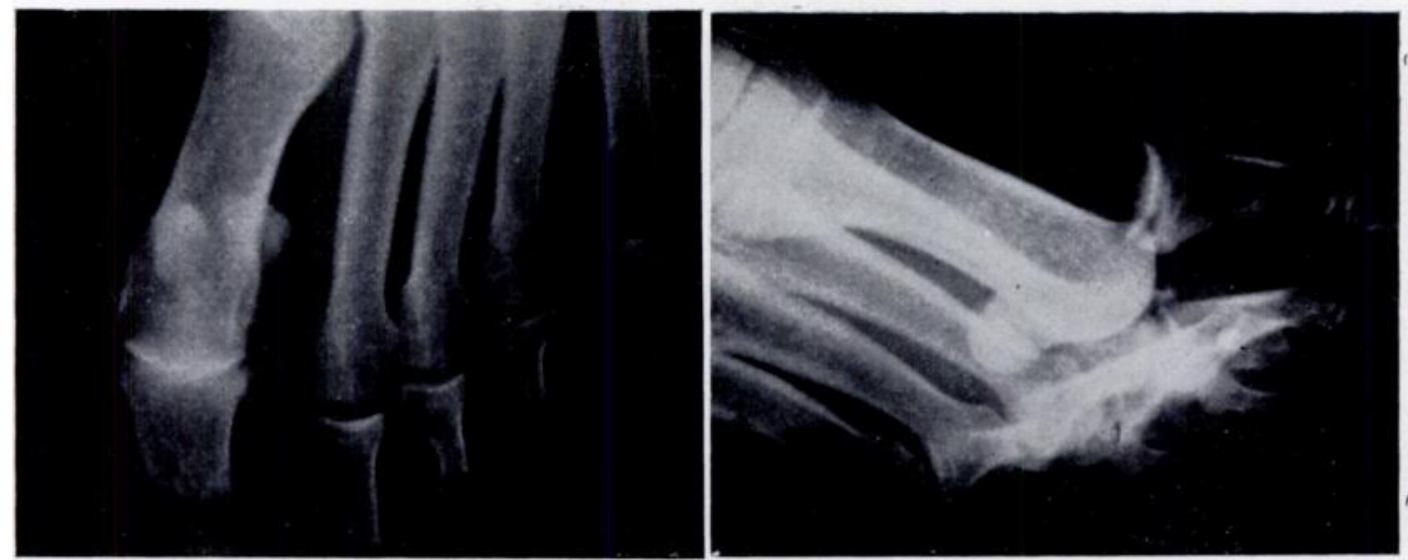

FIG. 1

Case 1-Antero-posterior and lateral radiographs of the dorsal dislocation. Note that the two sesamoids are not separated and not dorsally displaced.
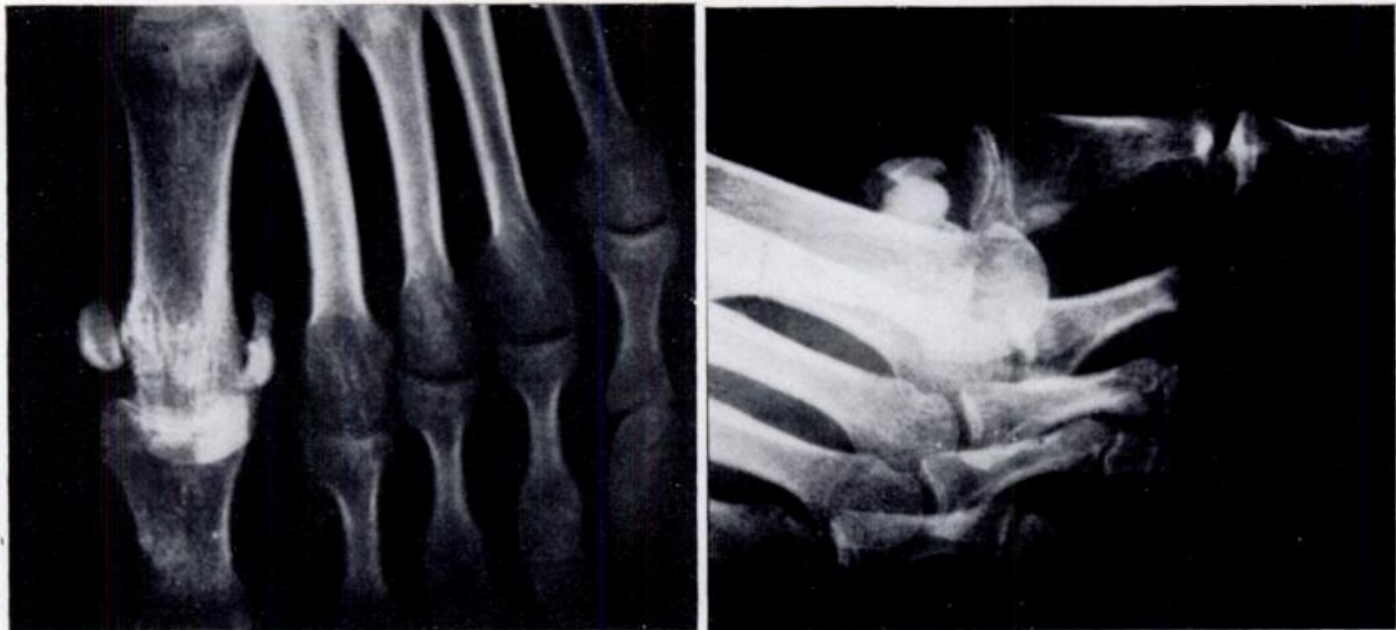

Fig. 2

Case 2-Antero-posterior and lateral radiographs showing the dorsal dislocation of the proximal phalanx. Note that the sesamoids are widely separated and lie dorsal to the neck of the metatarsal.
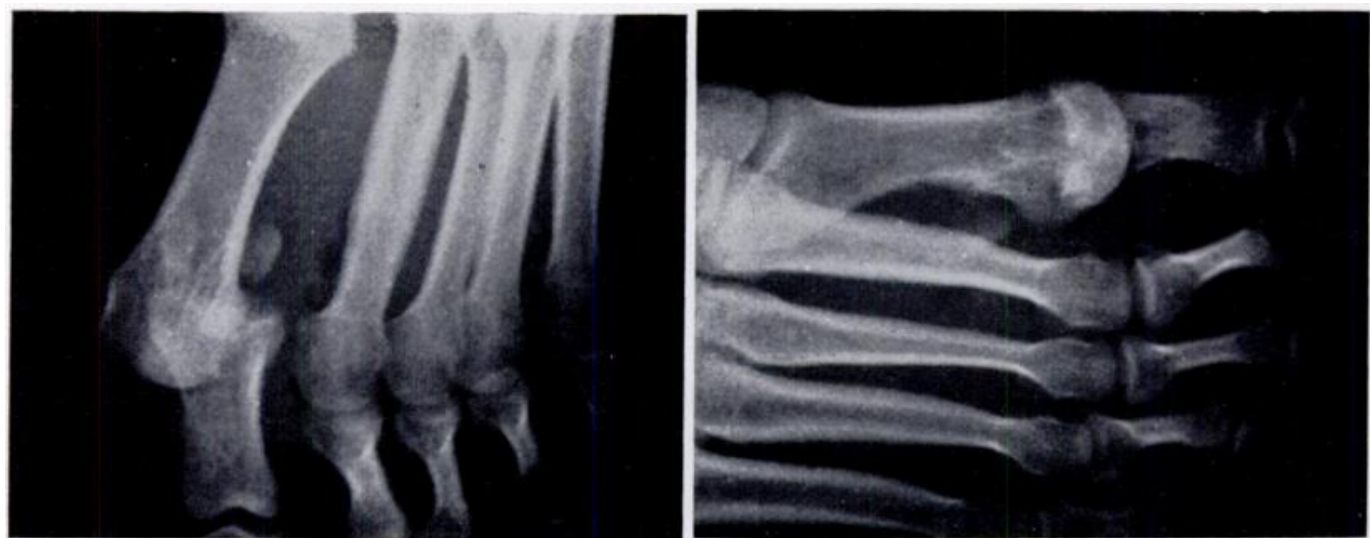

FIG. 3

Case 4-Antero-posterior and oblique radiographs taken three weeks afterinjury, showing the dislocation and the position of the sesamoids. Note the amount of overlap that has developed, making open reduction impossible. 
At operation, under general anaesthesia and in a bloodless field, a transverse plantar incision was made under the first metatarso-phalangeal joint. The head of the metatarsal was found just under the skin with the flexor tendons laterally. At this stage a further attempt was made to reduce the dislocation by traction and pressure, but again this failed. The flexor tendons were then retracted to one side, when it was noticed that the collateral ligaments were intact; this explained why the traction used was inadequate to separate the bones and complete the reduction. A lever was then placed dorsal to the head and close to its cartilaginous surface in order to hold back the fibrocartilaginous plate of the joint, and by pulling and levering, the dislocation was reduced. Firm attempts at redislocation failed.

The foot was immobilised for two weeks in a voluminous pressure dressing and the patient resumed full weight-bearing in three weeks. No problems have arisen during six years of follow-up.

Case 2, that of a man aged twenty-four injured in April
1970 (Fig. 2) and Case 3, that of a man aged twenty-one injured in August 1970, were similar to Case 1 and were successfully treated the same way.

Case 4-A man aged thirty-five years sustained multiple injuries in a car accident in June 1972 for which he was treated in an intensive care unit for three weeks. The dislocation was overlooked (Fig. 3). An attempt at closed reduction under general anaesthesia failed, as did open reduction. Adhesions and scarring of the region made it necessary to remove the proximal end of the first phalanx and change the operation to Keller's arthroplasty. The patient has had no problems since.

Postscript: Since this report was submitted for publication a paper reporting a similar case with similar conclusions has appeared in the American literature (Salmon, Gelberman and Huffer 1974).

\section{REFERENCES}

Gray (1967) Anatomy: Descriptive and Applied. Thirty-fourth edition, pp. 580-581. Edited by D. V. Davies with R. E. Coupland. London: Longmans.

Salamon, P. B., Gelberman, R. H., and Huffer, J. M. (1974) Dorsal dislocation of the metatarso phalangeal joint of the great toe. Journal of Bone and Joint Surgery, 56-A, 1073-1075. 